Irish Math. Soc. Bulletin

Number 69, Summer 2012, 47-56

ISSN 0791-5578

\title{
THE EQUATION OF TIME AND THE ANALEMMA
}

\author{
PETER LYNCH
}

\begin{abstract}
The Earth's progress around the Sun varies through the year. Combined with the tilt of the axis of rotation, this results in variations of the length of a solar day. The variations are encapsulated in the Equation of Time. A plot of altitude versus azimuth for the Sun at 12 noon local time through the year describes a figure-of-eight curve known as an analemma. By analysis of the observations, we find that the qualitative aspects of the analemma can be reproduced using just two sinusoidal components.
\end{abstract}

\section{INTRODUCTION}

An analemma is the curve obtained by plotting the position of the Sun, as viewed from a fixed location on Earth, at the same clock time each day for a year. If the Earth's orbit were perfectly circular and the axis of rotation were perpendicular to the plane of the orbit, the analemma would collapse to a fixed point. However, the orbit is elliptical and the axis tilted, and the analemma is a large figureof-eight. This has important consequences for the measurement of time.

On the East Pier in Dun Laoghaire there is an analemmatic sundial. The hour-points are on an ellipse, the horizontal projection of a circle parallel to the equator. The gnomon is formed by the observer, whose shadow falls on the ellipse, indicating the time. Three adjustments must be made to get mean time from sun-dial time. First, since Dun Laoghaire is six degrees, eight minutes west of Greenwich, 25 minutes must be added. Next, a seasonal correction must be made. This is read from a graph of the Equation of Time, conveniently plotted on a bronze plaque (Fig. 1). Finally, an extra hour must be added during Irish Summer Time. In this

2010 Mathematics Subject Classification. 85-01, 85A04.

Key words and phrases. Mean Time, Analemma.

Received on 10-8-2012; revised 24-8-12. 


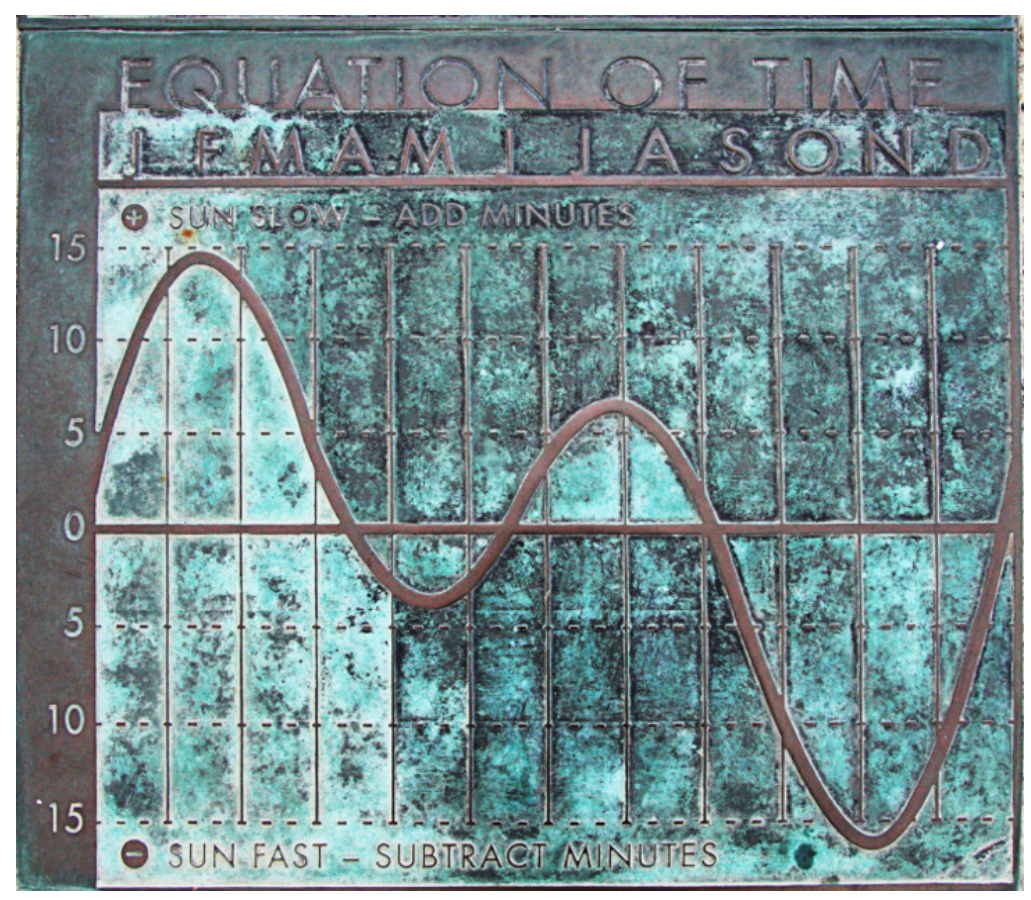

Figure 1. Bronze plaque indicating the Equation of Time, on the analemmatic sundial installed on the East Pier in Dun Laoghaire (Graph drawn by Capt. Owen M. Deignan. Photo: Peter Lynch).

paper, we will examine the Equation of Time and show how it can be expressed approximately in terms of two sinusoidal components.

\section{OBservations}

The position of the Sun in the sky, as seen from the Royal Observatory, Greenwich at 12:00 GMT each day for 2006, is available online [5. The position is specified by two angles, analogous to latitude and longitude: the altitude, or angle relative to the horizon and the azimuth, or angle relative to true north. A plot of altitude versus azimuth (Fig. 22) describes a figure-of-eight curve known as an analemma.

The apparent variation in the position of the Sun has been intensively studied by astronomers, and is well understood. It is the cause of variations in the length of a solar day and the difference between solar time and mean time. The variations are encapsulated in an expression called the Equation of Time (the term 'equation' is used here in a historical sense, meaning a correction or adjustment). The difference between mean and solar time can be predicted with 


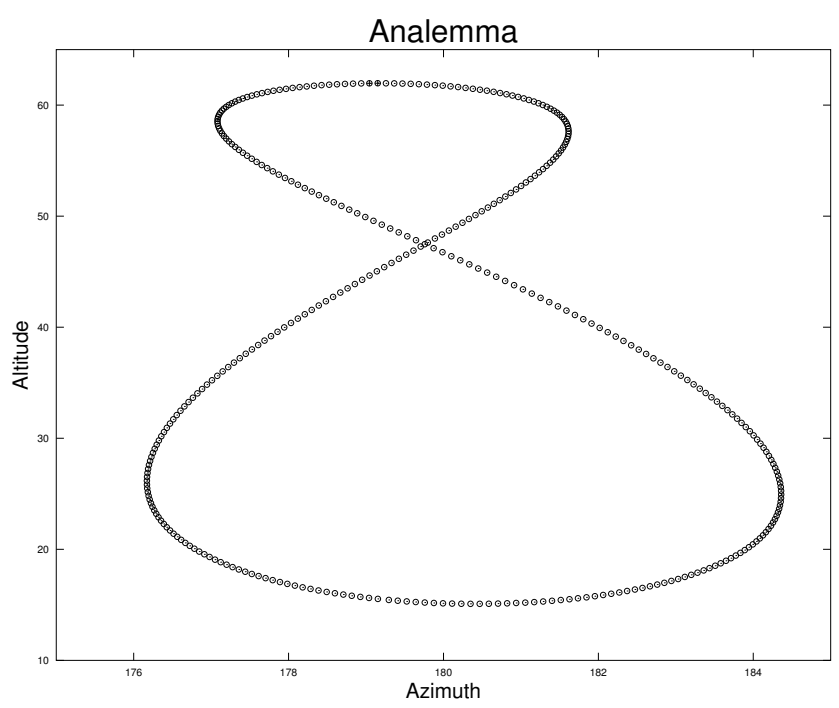

FiguRE 2. The analemma, based on observed values of the azimuth and altitude of the Sun at 12.00 GMT at the Royal Observatory, Greenwich for 2006 (see [5]). Note the unequal axes.

great precision. For example, [2] gives an algorithm that calculates the Equation of Time valid over a period of 6000 years, accurate to within three seconds. So, accurate estimation of the correction is not an issue. However, examination of the data shows that there are dominant components of the Equation of Time that call for explanation, and it is instructive and illuminating to examine these and explain them in terms of the main variations in the Earth's orbit. This is the goal of the present note.

A Fourier analysis of the altitude and azimuth for the year 2006 shows that only the first few components have appreciable amplitude. Fig. 3 (left panel) makes it clear that the altitude is dominated by the component with a period of a year; the Sun moves between the Tropics of Cancer and Capricorn in an essentially sinusoidal fashion. In Fig. 3 (right panel), the amplitudes of the coefficients of the transformed azimuth show that components 1 and 2 are dominant; component 3 is not negligible, but it is substantially smaller than the two main components. Thus, the principal variations in azimuth have periods of a year and a half year.

When the altitude is plotted against the azimuth, the figure-ofeight curve shown in Fig. 2 results. The pattern can be further broken down by taking the in-phase and quadrature elements of each 

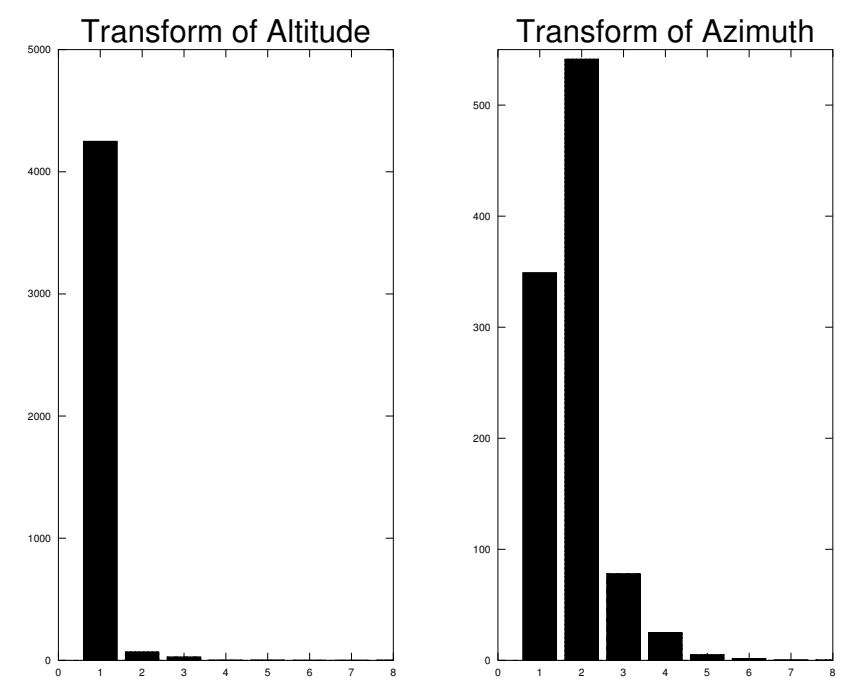

FiguRE 3. Magnitude of the Fourier components of altitude (left) and azimuth (right). Only the first 8 components are shown.

of the two components of the azimuth. Thus, for the annual component, we plot the part in phase with the altitude in Fig. 4(A) and the part orthogonal to altitude in Fig. 4(B). Similarly, the two parts of the semi-annual component of azimuth are plotted in Fig. $4(\mathrm{C})$ and Fig. 4(D).

The aim of the remainder of this paper is to explain the observed variations in terms of the characteristics of the orbit of the Earth. There are two main variations, the eccentricity of the Earth's elliptical orbit and the obliquity, or tilt of the axis relative to the ecliptic or plane of the orbit around the Sun. We will examine each in turn. We remark that highly accurate values for all the quantities and expressions that we consider are available in the astronomical literature. The present note is concerned with elucidating mechanisms rather than with precision.

\section{VARIATIONS DUE TO ELLIPTICITY OF THE ORBIT}

To a high degree of approximation, the Earth's orbit is a Keplerian ellipse. Perihelion in 2006 was on 4 January and aphelion on 3 July. The Earth rotates about its axis in a sidereal day and revolves about the Sun in a year, so the ratio of mean angular velocity of revolution $\varpi$ to that of rotation $\Omega$ is about $1 / 365$. 

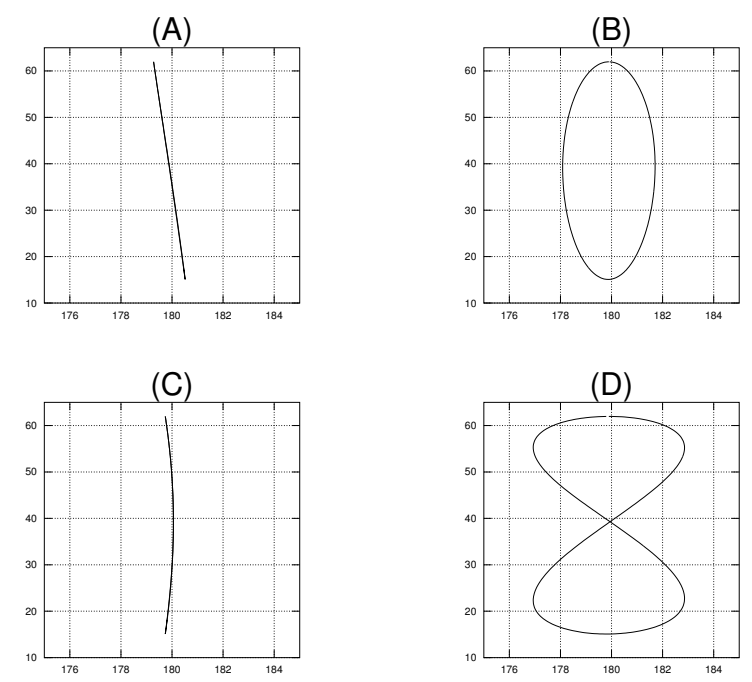

FiguRE 4. Lissajous components of the analemma. A: In-phase annual component; B: Quadrature annual component; C: In-phase semi-annual component; D: Quadrature semi-annual component.

By Kepler's Second Law, the angular momentum (per unit mass) $h=r^{2} \dot{\theta}$ is constant [6]. Here, $r$ is the distance between the Earth and Sun and $\theta$ is the 'true anomaly', the angle between the radius vector and the line from the Sun to the perihelion. Let $a$ be the semi-major axis and $e$ the eccentricity of the orbit. The perihelion and aphelion distances are respectively $r_{\mathrm{P}}=(1-e) a$ and $r_{\mathrm{A}}=(1+e) a$ and, if the angular velocities at these points are $\omega_{\mathrm{P}}=\dot{\theta}_{\mathrm{P}}$ and $\omega_{\mathrm{A}}=\dot{\theta}_{\mathrm{A}}$, we have

$$
h=(1-e)^{2} a^{2} \omega_{\mathrm{P}}=(1+e)^{2} a^{2} \omega_{\mathrm{A}} .
$$

Since the eccentricity is small ( $e \approx 0.0167$ ), we can take the mean angular velocity to be $\varpi=h / a^{2}$. Then

$$
\begin{aligned}
& \omega_{\mathrm{P}}=h /[(1-e) a]^{2} \approx(1+2 e) \varpi \\
& \omega_{\mathrm{A}}=h /[(1+e) a]^{2} \approx(1-2 e) \varpi
\end{aligned}
$$

As the Earth rotates and revolves, the Sun appears to revolve about it with angular velocity $\Omega-\omega$, so the difference between the rates at aphelion and perihelion is

$$
\left(\Omega-\omega_{\mathrm{A}}\right)-\left(\Omega-\omega_{\mathrm{P}}\right)=\left(\omega_{\mathrm{P}}-\omega_{\mathrm{A}}\right) \approx 4 e \varpi
$$

Thus, the fractional change is $4 e \varpi / \Omega$. This determines the length of a solar day, which may be shorter or longer than the mean day 
by an amount $2 e \varpi / \Omega \approx 9.15 \times 10^{-5} \mathrm{~s} \mathrm{~s}^{-1}$, or 7.9 seconds in a day. This is small, but it accumulates over a period of weeks or months.

The variation in the length of a solar day can be approximated by a sinusoidal wave with amplitude $2 e \varpi / \Omega$, varying on an annual cycle:

$$
\Delta_{\mathrm{ECC}}=\frac{2 e \varpi}{\Omega} \cos M_{1}
$$

where $M_{1}=2 \pi\left(D-D_{\mathrm{P}}\right) / 365$ with $D$ the day number and $D_{\mathrm{P}}$ the date of perihelion. Eccentricity causes a lengthening of the solar day at perihelion (near mid-winter) and a shortening at aphelion (near mid-summer), and $\Delta_{\mathrm{ECC}}$ is the amount that must be added to correct solar time for the effect of the Earth's elliptic orbit.

\section{VARIATIONS DUE TO OBLIQUity OF THE ORBIT}

Let us now disregard the eccentricity temporarily, and assume that the Earth's orbit is circular. If the axis of rotation were perpendicular to the ecliptic, or plane of the Earth's orbit around the Sun, each day would be the same length. The Earth would advance by about $1^{\circ}$ during the course of a sidereal day, so a solar day would be longer by a factor of about $\frac{1}{360}$, or about 4 minutes. However, the equatorial plane of Earth is tilted to the ecliptic by an angle $\epsilon \approx 23.44^{\circ}$, called the obliquity. So, the ecliptic plane cuts the earth in a great circle that makes an angle $\epsilon$ with the equator at the two points where they intersect. These points correspond to the equinoxes.

Let us also disregard the Earth's rotation momentarily; effectively, we are taking a stroboscopic view with a frequency $\Omega$. Then, during the course of a year, the Sun will trace out the great circle at a constant rate. The equation for the great circle is an elementary geometrical exercise; the latitude $(\phi)$ and longitude $(\lambda)$ are related by

$$
\tan \phi=\tan \epsilon \sin \left(\lambda-\lambda_{0}\right)
$$

where $\lambda_{0}$ corresponds to the vernal equinox. For simplicity, we set $\lambda_{0}=0$. Eqn. (2) corresponds to one of Napier's rules for right spherical triangles; see [8, p. 888]. Differentiating (2), we get

$$
\frac{\mathrm{d} \phi}{\mathrm{d} \lambda}=\frac{\tan \epsilon \cos \lambda}{1+\tan ^{2} \epsilon \sin ^{2} \lambda} .
$$

The progress of the Sun along the trajectory (2) is constant, but the change in longitude, which determines the time, is not. If we 
consider a small step $\mathrm{d} \sigma$ along the great circle, the spherical metric gives $\mathrm{d} \sigma^{2}=\cos ^{2} \phi \mathrm{d} \lambda^{2}+\mathrm{d} \phi^{2}$, so the change in $\lambda$ with respect to $\sigma$ is

$$
\frac{\mathrm{d} \lambda}{\mathrm{d} \sigma}=\left(1+\tan ^{2} \epsilon \sin ^{2} \lambda\right) \cos \epsilon .
$$

The extreme values follow immediately. For small or moderate values of the obliquity $\epsilon$, they are:

$$
\begin{array}{ll}
\text { Equinoxes }(\lambda=0, \pi): & \frac{\mathrm{d} \lambda}{\mathrm{d} \sigma}=\cos \epsilon \approx 1-\frac{1}{2} \epsilon^{2} \\
\text { Solstices }\left(\lambda=\frac{\pi}{2}, \frac{3 \pi}{2}\right): & \frac{\mathrm{d} \lambda}{\mathrm{d} \sigma}=\sec \epsilon \approx 1+\frac{1}{2} \epsilon^{2}
\end{array}
$$

Thus, the solar day may be shorter or longer than the mean day by an amount $\frac{1}{2} \epsilon^{2} \varpi / \Omega \approx 2.29 \times 10^{-4} \mathrm{~s} \mathrm{~s}^{-1}$, or 19.8 seconds in a day. We note that [3] gives a more accurate expression where the factor $\frac{1}{2} \epsilon^{2}$ is replaced by $2 \tan ^{2} \frac{\epsilon}{2}$. However, our concern here is less with precision and more with simplicity.

The variation in the length of a solar day can be approximated by a sinusoidal wave with amplitude $\frac{1}{2} \epsilon^{2} \varpi / \Omega$, varying on a semi-annual cycle:

$$
\Delta_{\mathrm{OBL}}=\frac{\epsilon^{2} \varpi}{2 \Omega} \cos 2 M_{2}
$$

where $M_{2}=2 \pi\left(D-D_{\mathrm{W}}\right) / 365$ with $D$ the day number and $D_{\mathrm{W}}$ the date of the winter solstice (day 355 in 2006). Obliquity causes a lengthening of the solar day at the equinoxes and a shortening at the solstices, and $\Delta_{\mathrm{OBL}}$ is the amount that must be added to correct solar time for its effect.

\section{The Equation of Time}

We now combine the effects of eccentricity (11) and obliquity (3) to get the total difference between solar and mean time, $\Delta=\Delta_{\mathrm{ECC}}+$ $\Delta_{\mathrm{OBL}}$. To calculate the accumulated difference, this must be integrated, to give

$$
\begin{aligned}
E & =\frac{2 e \varpi}{\Omega} \sin M_{1}+\frac{\epsilon^{2} \varpi}{4 \Omega} \sin 2 M_{2} \\
& =7.9 \sin \left(\frac{2 \pi(D-3)}{365}\right)+9.9 \sin 2\left(\frac{2 \pi(D-355)}{365}\right) .
\end{aligned}
$$




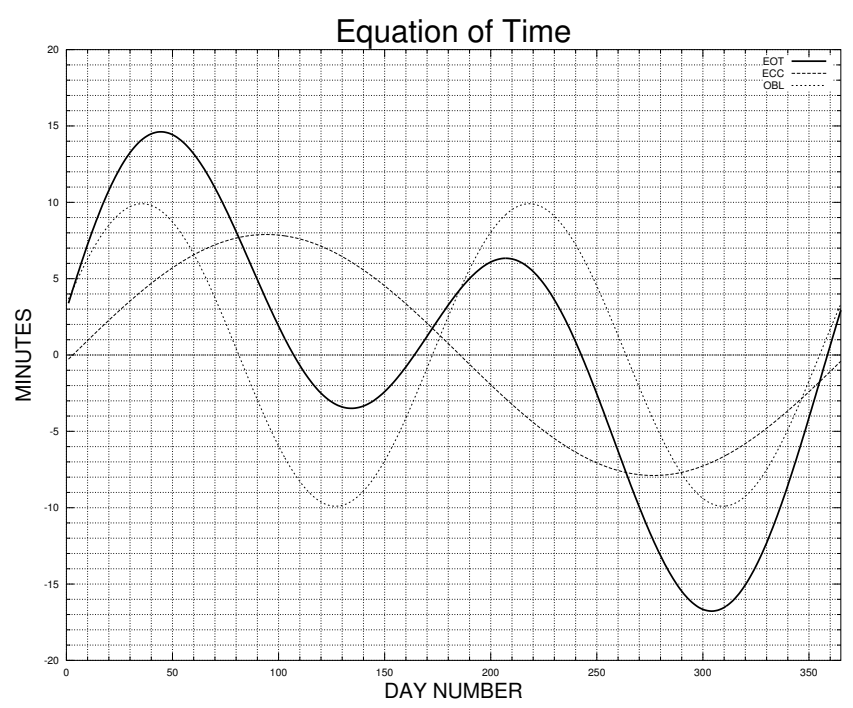

Figure 5. Equation of Time (in minutes) as a function of the day number, computed using (4). The components due to eccentricity (dashed) and obliquity (dotted) are shown. This is the correction that must be added to solar time to get mean time.

This gives the difference between the mean and solar time in minutes. It varies by about 15 minutes in both directions. The approximate curve, together with the two components, due to eccentricity and obliquity, are shown in Fig. 5. There is good qualitative agreement with the curve in Fig. 1 .

To construct an approximation to the analemma, we convert time in minutes given by (4) to degrees longitude by dividing by 4 and adding $180^{\circ}$. The approximate and observed curves are plotted in Fig. 6. We see that the main features of the observed pattern are replicated, but there are significant differences. These discrepancies can be reduced by including higher terms. A very precise, but more complicated, description of the Equation of Time is given in [2].

\section{Discussion}

The difference between mean time and solar time is expressed as the Equation of Time. Fourier analysis of the observations at the Royal Observatory in Greenwich shows that the variations in the Sun's noontime position are dominated by the first few Fourier coefficients. This allows us to approximate the Equation of Time by two 


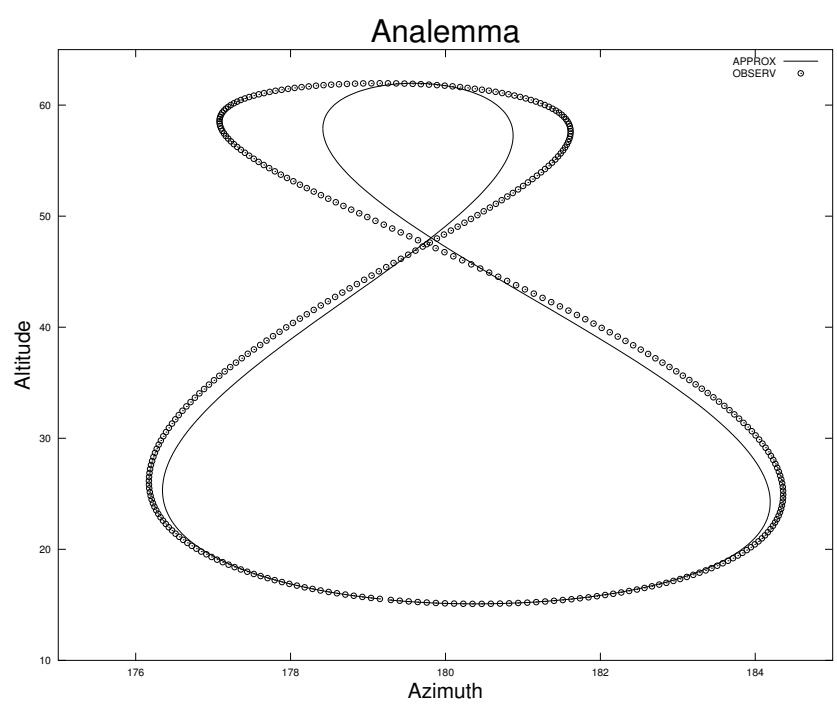

Figure 6. Solid line: analemma based on the approximate Equation of Time (4). Dotted line: analemma based on observations, as in Fig. 2.

sinusoidal components, with periods of a year and a half year. The curve that results from plotting the resulting approximation against solar altitude is qualitatively similar to the observed analemma.

The analemma has many applications. It can be used to estimate the time and azimuth of sunrise and sunset, and to explain the occurrence of latest sunset some days before the winter solstice and the earliest sunrise some days later. Many geosynchronous, but not geostationary, satellites move on analemmatic curves, and the control systems for the paraboloidal dishes used to track them must compute these curves to ensure optimum communications. Finally, the Equation of Time is important in many scientific and engineering contexts. It is used for the design of solar trackers and heliostats, vital for harnessing solar energy.

If more precise approximations of the Equation of Time are required, they are available in [2]. Practical information on the construction of analemmatic sundials is presented in [1] and [7], and a program to compute the design for a given location is given in [4].

\section{REFERENCES}

[1] Budd, C. J. and C. J. Sangwin, 2001: Mathematics Galore. Oxford Univ. Press., 254pp. 
[2] Hughes, D. W., B. D. Yallop and C. Y. Hohenkerk, 1989: The equation of time. Mon. Not. Roy. Astr. Soc., 238, 1529-1535.

[3] Milne, R. M., 1921: Note on the Equation of Time. Math. Gaz., 10, 372-375.

[4] Pruss, Alexander R., 2011: Analemmatic Sundial PDF Generator (program to design an analemmatic sundial, given the latitude, longitude and timezone). http://analemmatic.sourceforge.net/cgi-bin/sundial.pl

[5] RGO, 2006: Azimuth and altitude of Sun at Royal Observatory, Greenwich, 2006. Available online at http://en.wikipedia.org/wiki/File:Analemma_Earth.png

[6] Roy, A. E., 2005: Orbital Motion. 4th Edn. Inst. of Phys. Publ. 592pp.

[7] Sangwin, Chris and Chris Budd, 2000: Analemmatic sundials: how to build one and why they work. Plus Magazine, Issue 11, June 2000. http://plus. maths.org/content/os/issue11/features/sundials/index

[8] Vallado, D. A., 2001: Fundamentals of Astrodynamics and Applications. Published jointly by Microcosm Press, Calif. and Kluwer Acad. Press Dordrecht, 958pp.

Peter Lynch is Professor of Meteorology at UCD. His interests include dynamic meteorology, numerical weather prediction, Hamiltonian mechanics and the history of meteorology. He writes an occasional mathematics column That's Maths in the Irish Times; see his blog at http://thatsmaths.com.

School of Mathematical Sciences, University College Dublin E-mail address: Peter.Lynch@ucd.ie 